\title{
Pulmonary artery intimal sarcoma misdiagnosed as pulmonary embolism: A case report
}

\author{
SHUYAN JIANG ${ }^{1-3}$, JIAXIN LI ${ }^{2,3}$, QINGSHI ZENG ${ }^{3}$ and JIEXIAN LIANG ${ }^{3}$ \\ ${ }^{1}$ Department of Anesthesiology, Guangdong No.2 Provincial People's Hospital, Guangzhou, Guangdong 510317; \\ ${ }^{2}$ Postgraduate Institute, Southern Medical University, Guangzhou, Guangdong 510515; ${ }^{3}$ Division of Anesthesiology, \\ Department of Cardiovascular Surgery, Guangdong Institute of Cardiovascular Diseases, Guangdong General Hospital, \\ Guangdong Academy of Medical Science, Guangzhou, Guangdong 510080, P.R. China
}

Received March 23, 2015; Accepted June 27, 2016

DOI: $10.3892 / \mathrm{ol} .2017 .5775$

\begin{abstract}
Intimal sarcoma of the pulmonary artery is rare, but false diagnosis of this sarcoma as pulmonary embolism is not infrequent. The present study reports a case of pulmonary artery intimal sarcoma misdiagnosed as pulmonary artery embolism in a 37-year-old female patient. The patient was admitted to the cardiac intensive care unit of Guangdong General Hospital (Guangzhou, China) with the complaint of progressive exertional dyspnea over the past two years. Multi-slice spiral computed tomography, transthoracic echocardiography, right-heart catheterization and cardiac magnetic resonance imaging revealed mimicking severe pulmonary embolism in the pulmonary trunk and right pulmonary artery, with symptoms including chest pain, cough and breathing difficulties. In addition, positron emission tomography-computed tomography results did not identify increased $18 \mathrm{~F}$-fluorodeoxyglucose uptake and failed to distinguish whether the mass was a thrombus or a malignancy. The patient was diagnosed with pulmonary embolism and a subcutaneous injection of 5,000 AxaIU enoxaparin sodium (100 AXAIU/kg) was administered every $12 \mathrm{~h}$, but no improvement was achieved after 5 days of treatment. Finally, pulmonary endarterectomy was performed to relieve the worsening clinical symptoms. The clinicopathological diagnosis was pulmonary artery intimal sarcoma with poor clinical outcome. For this type of
\end{abstract}

Correspondence to: Professor Jiexian Liang, Division of Anesthesiology, Department of Cardiovascular Surgery, Guangdong Institute of Cardiovascular Diseases, Guangdong General Hospital, Guangdong Academy of Medical Science, 96 Dongchuan Road, Guangzhou, Guangdong 510080, P.R. China

E-mail: lijessy@msn.com

Abbreviations: ECMO, extracorporeal membrane oxygenation; PEA, pulmonary endarterectomy; PET-CT, positron emission tomography-computed tomography; MRI, magnatic resonance imaging; SMA, smooth muscle actin

Key words: pulmonary artery intimal sarcoma, pulmonary embolism, difficult diagnosis, poor prognosis tumor with fatal prognosis, early and correct diagnosis may lead to appropriate intervention and prolong survival.

\section{Introduction}

Since Moritz Mandelstamm first described intimal sarcoma of the pulmonary artery in 1923 (1), this tumor has been an uncommon but aggressive malignant tumor among the pulmonary artery, and may develop a fatal course leading to heart failure without treatment $(2,3)$. The incidence is reported to be $0.001 \%$, but this incidence appears to be inaccurate due to the challenging (4). Pulmonary artery intimal sarcoma has similar clinical manifestations to pulmonary embolism (including chest pain, difficulty breathing, cough, chest tightness, oedema and hemoptysis), which leads to an initially false diagnosis and delayed interventions (5). Percutaneous intravascular biopsy using a bronchoscopy forceps or ultrasound-guided transbronchial needle aspiration may obtain an early definitive diagnosis $(2,3)$. The prognosis of this tumor is extremely poor, with a survival time of 12-18 months subsequent to the appearance of clinical symptoms (6). Surgical resection is the main treatment to prolong survival, which includes pulmonary endarterectomy (PEA), lobectomy and pneumonectomy (7). Combination doxorubicin and ifosfamide as first-line chemotherapy has been reportedto be transiently effective in the inhibition of rapid enlargement of the tumor. However, the effects of adjuvant radiation, radiofrequency ablation and chemotherapy subsequent to surgery remain unclear, and further studies are warranted (5).

\section{Case report}

A 37-year-old woman was admitted to Guangdong General Hospital (Guangzhou, China) on December 25, 2014 with the complaint of exertional dyspnea of a two-year duration, which had been worsening for 5 days. The patient was classified as class III on the New York Heart Association classification, and the patient was relatively hemodynamically stable (8). On physical examination, the pulse rate was 98 beats/min (normal range, $60-100$ beats $/ \mathrm{min}$ ), with blood pressure 130/80 mmHg (normal range, 90-139/60-89 mmHg). The patient demonstrated tachypnea, with 24 breaths/min 

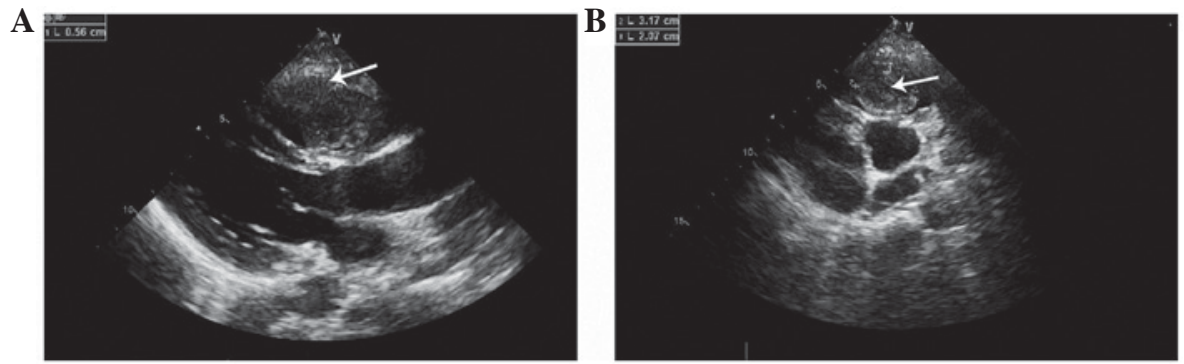

Figure 1. Transthoracic echocardiography results. (A) A large mass was identified in the right ventricular outflow tract in the left ventricular long axis view. (B) In the aortic root short axis view, a mass $(3.17 \times 2.07 \mathrm{~cm})$ was identified in the initial segment of the pulmonary trunk.
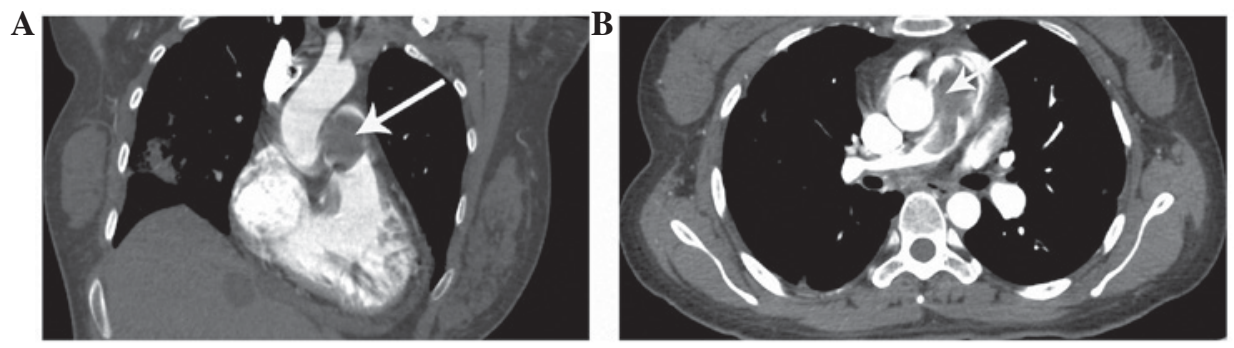

Figure 2. Chest contrast-enhanced computed tomography results. (A) The intraluminal defections in the main pulmonary artery. (B) The intraluminal defections extended to the right pulmonary artery.

(normal range, 12-18 breaths/min), and her oxygen saturation was $94 \%$ at rest (normal range, $95-100 \%$ ), with no clubbing or cyanosis. Cardiac auscultation revealed a systolic grade 3 murmur at the upper left sternal border. The laboratory results revealed no evident abnormalities, with the exception of the N-terminal pro-B-type natriuretic peptide level, which was $5,382 \mathrm{pg} / \mathrm{ml}$ (normal range for females $<46$ years, $10-140 \mathrm{pg} / \mathrm{ml})$. The electrocardiogram and chest radiography were normal. Transthoracic echocardiography demonstrated that the right ventricle was enlarged to $52 \mathrm{~mm}$, and a large mass $3.17 \times 2.07 \mathrm{~cm}$ in size was identified in initial segment of pulmonary trunk (Fig. 1). The color Doppler ultrasound detected mild tricuspid regurgitation. The pulmonary arterial pressure was estimated as $76 \mathrm{mmHg}$ (normal range, $18-25 \mathrm{mmHg}$ ), which indicates severe pulmonary hypertension. The chest contrast-enhanced computed tomography detected intraluminal defects in the main pulmonary artery, extending to the right pulmonary artery (Fig. 2). Cardiac catheterization showed a defect in repletion at the level of the pulmonary trunk, which almost occluded the systolic blood flow in the right upper pulmonary artery. In addition, cardiac magnetic resonance imaging (MRI) findings also revealed the thrombotic feature (Fig. 3). Positron emission tomography-computed tomography (PET-CT) was performed to distinguish whether the pulmonary artery mass was a thrombus or neoplasm. PET-CT did not find evident activity in the region of the pulmonary artery or neoplasm metastasis signals (Fig. 4). The patient was diagnosed with pulmonary artery embolism, and a subcutaneous injection of 5,000 AxaIU enoxaparin sodium (100 AxaIU/kg) was administered every $12 \mathrm{~h}$, but no improvement was achieved after 5 days of treatment. Finally, 2-year progressive dyspnea and 15-day right heart failure resulted in the decision to perform a surgical approach to relieve the obstruction of

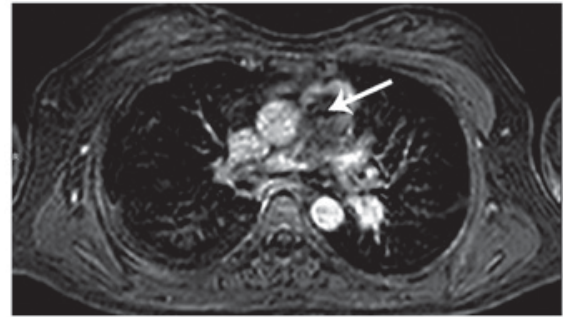

Figure 3. Cardiac magnetic resonance imaging findings also revealed thrombotic features in the main pulmonary artery, extending to the right pulmonary artery. The arrow indicates a thrombus-like mass.

the pulmonary artery and to define the diagnosis. PEA was performed. The gross appearance of the tissue was a yellow grey dendritic-like mass, and intraoperative frozen biopsy suggested sarcoma. Finally, extracorporeal membrane oxygenation (ECMO) was used to stabilize the vital status of the patient.

Histopathology revealed the tissue to be myxoid sarcoma, with spindle cells and mitotic figures easily identified. Microscopically, the tissue mesenchyme was evidently mucoid, tumor cells were pleomorphic and nuclei were a rounded, ovate or fusiform in shape. The single tumor giant cells were sporadic and certain tumor cells were observed with a growth pattern surrounding the small vessel. Certain tumor cells were observed with a growth pattern surrounding the small vessel (Fig. 5). Immunohistochemical analysis was performed according to Neuville et al (9). Immunohistochemical findings showed focal positivity for vimentin (antibody dilution, 1:1,000; 2128; Dako, Glostrup, Denmark), and slight positivity for desmin (antibody dilution, 1:200; 7074; Dako) and smooth muscle actin (SMA; antibody dilution, 1:200; 9102; Dako), while CD34 (antibody dilution, 1:400; 9204; Dako) 


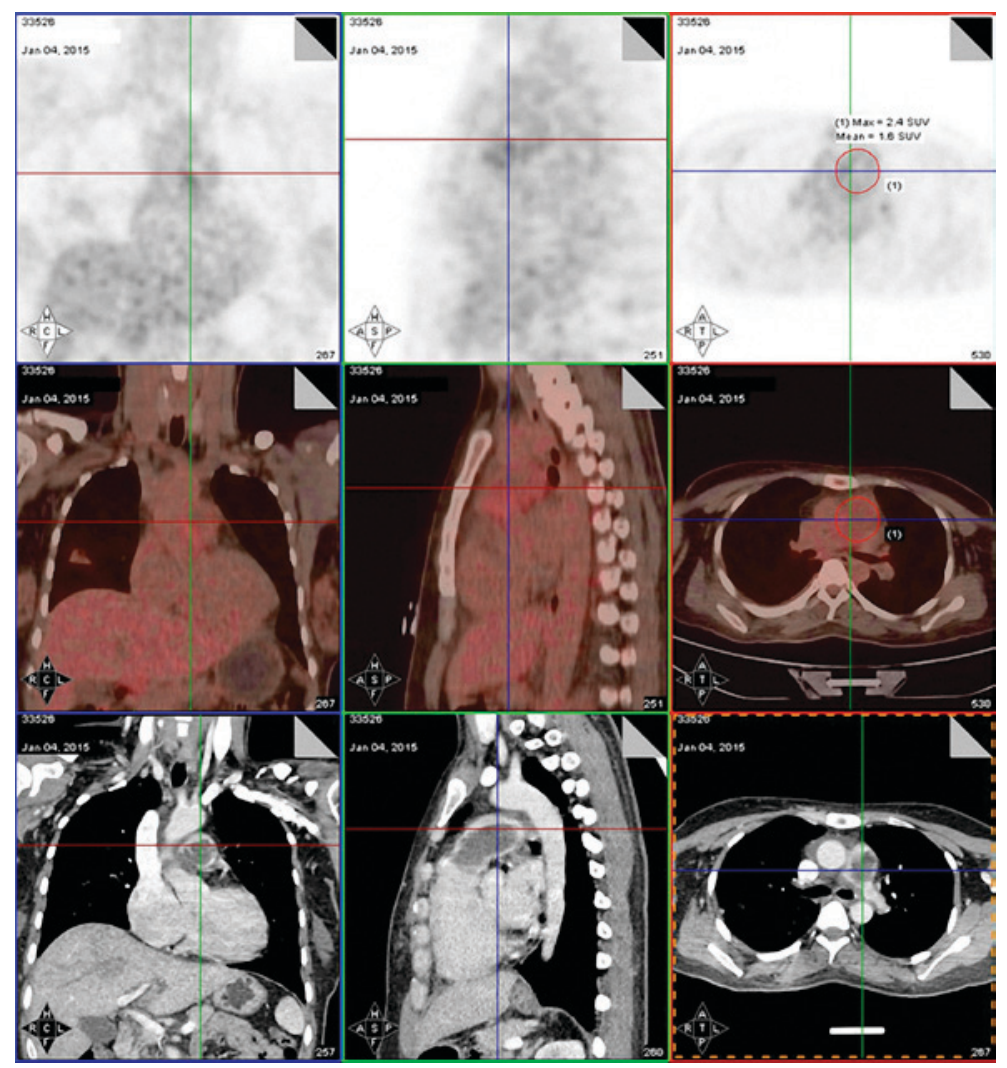

Figure 4. Positron emission tomography-computed tomography did not find evident abnormal metabolic active locations in the region of the pulmonary artery and neoplasm metastasis signals. First column, intraluminal defections in the right pulmonary artery at the coronal plane. Second column, intraluminal defections in the right pulmonary artery at the sagital plane. Third column, intraluminal defections int he right pulmonary artery at the axial plane.
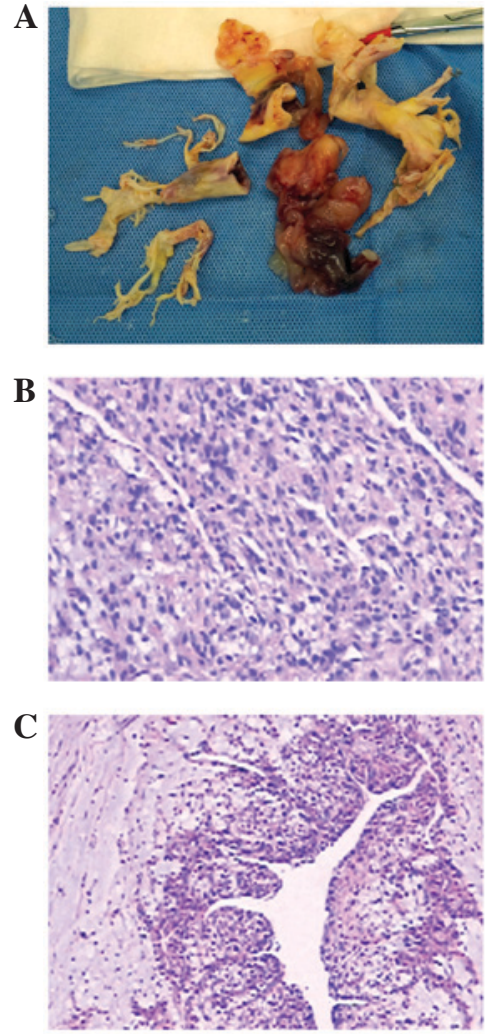

Figure 5. (A) Gross pathology of the tumor. (B and C) Histopathology revealed a myxoid sarcoma in which tumor cells were pleomorphic and nuclei were rounded, ovate and fusiform in shape. Certain tumor cells were shown with a growth pattern surrounding the small vessel. (B) Original magnification, $\mathrm{x} 100$. (C) Original magnification, $\mathrm{x} 400$. and S-100 protein (antibody dilution, 1:500; 4036; Dako) were not expressed. In addition, fluorescence in situ hybridization for MDM2 was performed on paraffin-embedded tissues according to Weaver et al (10). MDM2 is a frequent finding in intimal sarcomas of the pulmonary artery. Amplification of MDM2 was considered positive in the present case (11).

ECMO was withdrawn from the patient 6 days subsequent to resection and the vital signs of the patient were stable. The patient and family members rejected additional chemotherapy and radiotherapy. At the last follow-up on March 11, 2015, the patient had been free of relapse for $>2$ months under clinical monitoring.

\section{Discussion}

Intimal sarcoma is an uncommon mesenchymal tumor, and it may originate from the great arteries, such as the pulmonary artery. Intimal sarcoma of the pulmonary artery is usually located in the center of the pulmonary trunk and extends into the right or left pulmonary artery, but retrograde extension has been reported (6). The age of the patients diagnosed with this fatal tumor ranges between 13 and 89 years (12) Gender predominance in the incidence of intimal sarcoma is controversial and has been reported as more common in women by Hsing et al (5). The true incidence is challenging to estimate due to the difficulty of diagnosis from current imaging technology. Intimal sarcoma of the pulmonary artery demonstrates an insidious development and firm diagnosis is always based on autopsy and postoperative histopathology. However, 
Hu et al (13) described a case in which a diagnosis of intimal sarcoma of the pulmonary artery was successfully made by percutaneous transcatheter aspiration. This is one way to diagnose intimal sarcoma prior to median sternotomy, irrespective of possible complications. Gadolinium-enhanced magnetic resonance has been reported to be sensitive for differentiating thrombi from tumors, since this imaging modality may detect the depth of defects in repletion in the lumen $(14,15)$; however, in the present case, cardiac MRI did not provide evident tumor signals. Regularly, PET-CT is recommended to detect malignant neoplasms and distant metastasis, but the PET-CT results of the present patient did not identify increased 18F-fluorodeoxyglucose (18F-FDG) uptake and failed to differentiate whether the mass was a thrombus or a malignancy. A previous study has revealed that poor $18 \mathrm{~F}$-fluorodeoxyglucose uptake may account for the misdiagnosis (16). However, another study reports that FDP-PET has been useful for diagnosis (17). Intimal sarcoma of the pulmonary artery has no specific symptoms at an early stage and has been easily misdiagnosed as pulmonary thromboembolism due to the resemblance in clinical features, such as dyspnea, pleuritic pain, cough and hemoptysis (18). Intimal sarcoma of the pulmonary artery demonstrates an extremely poor outcome, due to delayed intervention and easy recurrence. The median survival time of patients without surgical resection is only 1.5 months, and 10 months with resection (12). Surgical resection is the main treatment of this tumor (7). The effects of adjunctive chemotherapy and radiotherapy remain uncertain and standard chemotherapy regimens have not been unified. However, intimal sarcoma of the pulmonary artery has been reported to be chemosensitive in two cases $(19,20)$. Due to the extremely poor outcome, radiotherapy and chemotherapy appear to be methods to consider for inoperable patients. A diagnosis of pulmonary artery intimal sarcoma should be suspected, even if the imaging technology has not revealed the information of the tumor, particularly when regular anticoagulant therapy fails. Earlier diagnosis and resection may carry an improved prognosis.

\section{References}

1. Mandelstamm M. About primary neoplasms of the heart. Virchows Arch 245: 43-54, 1923.

2. Blackmon SH, Rice DC, Correa AM, Mehran R, Putnam JB, Smythe WR, Walkes JC, Walsh GL, Moran C, Singh H, et al: Management of primary pulmonary artery sarcomas. Ann Thorac Surg 87: 977-984, 2009.

3. Mussot S, Ghigna MR, Mercier O, Fabre D, Fadel E, Le Cesne A, Simonneau G and Dartevelle P: Retrospective institutional study of 31 patients treated for pulmonary artery sarcoma. Eur J Cardiothorac Surg 43: 787-793, 2013.

4. Vaideeswar P and Pillai R: Pulmonary arterial intimal sarcoma with retrograde extension: Report of a case and review of literature. Indian J Pathol Microbiol 56: 47-50, 2013.
5. Hsing JM, Thakkar SG, Borden EC and Budd GT: Intimal pulmonary artery sarcoma presenting as dyspnea: Case report. Int Semin Surg Oncol 4: 14, 2007.

6. Ozbek C, Emrecan B, Calli AO and Gurbuz A: Intimal sarcoma of the pulmonary artery with retrograde extension into the pulmonic valve and right ventricle. Tex Heart Inst J 34: 119-121, 2007.

7. Mayer E, Kriegsmann J, Gaumann A, Kauczor HU, Dahm M, Hake U, Schmid FX and Oelert H: Surgical treatment of pulmonary artery sarcoma. J Thorac Cardiovasc Surg 121: 77-82.

8. Dolgin M (ed): Nomenclature and Criteria for Diagnosis of Diseases of the Heart and Great Vessels. Little Brown, London, UK, 1994.

9. Neuville A, Collin F, Bruneval P, Parrens M, Thivolet F, Gomez-Brouchet A, Terrier P, de Montpreville VT, Le Gall F, Hostein I, et al: Intimal Sarcoma Is the Most Frequent Primary Cardiac Sarcoma: Clinicopathologic and Molecular Retrospective Analysis of 100 Primary Cardiac Sarcomas. Am J Sugr Pathol 38: 461-469, 2014.

10. Weaver J, Downs-Kelly E, Goldblum JR, Turner S, Kulkarni S, Tubbs RR, Rubin BP and Skacel M: Fluorescence in situ hybridization for MDM2 gene amplification as a diagnostic tool in lipomatous neoplasms. Mod Pathol 21: 943-949, 2008.

11. Zhang H, Macdonald WD, Erickson-Johnson M, Wang X, Jenkins RB and Oliveira AM: Cytogenetic and molecular cytogenetic findings of intimal sarcoma. Cancer Genet Cytogenet 179: 149-149, 2007.

12. Alsoufi B, Slater M, Smith PP, Karamlou T, Mansoor A and Ravichandran P: Pulmonary artery sarcoma mimicking massive pulmonary embolus: A case report. Asian Cardiovasc Thorac Ann 14: e71-e73, 2006.

13. Hu W, Xie Y and Zhang DD: Pulmonary artery intimal sarcoma diagnosed by percutaneous transcatheter aspiration. Chin Med J (Engl) 126: 1590-1591, 2013.

14. Viana-Tejedor A, Mariño-Enríquez A, Sánchez-Recalde A and López-Sendón JL: Intimal sarcoma of the pulmonary artery: Diagnostic value of different imaging techniques. Rev Esp Cardiol 61: 1363-1365, 2008 (In English, Spanish).

15. Rafal RB, Nichols JN and Markisz JA: Pulmonary artery sarcoma: Diagnosis and postoperative follow-up with gadolinium-diethylenetriamine pentaacetic acid-enhanced magnetic resonance imaging. Mayo Clin Proc 70: 173-176, 1995.

16. Lee DH, Jung TE, Lee JH, Shin DG, Park WJ and Choi JH: Pulmonary artery intimal sarcoma: Poor 18F-fluorodeoxyglucose uptake in positron emission computed tomography. J Cardiothorac Surg 8: 40, 2013.

17. Ote EL, Oriuchi N, Miyashita G, Paudyal B, Ishikita T, Arisaka Y, Higuchi T, Hirato J and Endo K: Pulmonary artery intimal sarcoma: The role of ${ }^{18} \mathrm{~F}$-fluorodeoxyglucose positron emission tomography in monitoring response to treatment. Jpn J Radiol 29: 279-282, 2011.

18. Wong HH, Gounaris I, McCormack A, Berman M, Davidson D, Horan G, Pepke-Zaba J, Jenkins D, Earl HM and Hatcher HM: Presentation and management of pulmonary artery sarcoma. Clin Sarcoma Res 5: 3, 2015 .

19. Uchida A, Tabata M, Kiura K, Tanimoto Y, Kanehiro A, Aoe M, Ohohara N, Ueoka H and Tanimoto M: Successful treatment of pulmonary artery sarcoma by a two-drug combination chemotherapy consisting of ifosfamide and epirubicin. Jpn J Clin Oncol 35: 417-419, 2005

20. Xu Y, Wang K, Geng Y, Shao Y and Yin Y: A case of intimal sarcoma of the pulmonary artery successfully treated with chemotherapy. Int J Clin Oncol 17: 522-527, 2012. 